\title{
A Rare Location: A Giant Mesenteric Lipoma
}

Mehmet ÖZER ${ }^{1}$, Serap ULUSOY ${ }^{1}$, Ömer PARLAK²

${ }^{1}$ Department of General Surgery, Ankara Atatürk Training and Research Hospital, Ankara, Turkey.

${ }^{2}$ Department of General Surgery, Faculty of Medicine, Ankara Yıldırım Beyazıt University, Ankara, Turkey.

\section{ABSTRACT}

Lipomas are benign soft tissue tumors. They can rarely be mesenterically located and symptomatic. It is very important to conduct the differential diagnosis of large intra-abdominal mass lesions. Radiological imaging techniques are often used for diagnosis, but when these techniques are not sufficient or when it becomes obligatory to cure symptoms of the lesion, surgical excision should be performedThis study aimed to present a patient with complaints and symptoms of an intraabdominal giant mass lesion, the pathological diagnosis of which the revealed a giant mesenteric lipoma.

Key words: Mesenteric lipoma, giant lipoma, abdominal mass

\section{INTRODUCTION}

Lipomas are benign soft tissue tumors. They are the most common soft tissue tumors of adult age. They are commonly solitary, mobile, painless, and slow-growing lesions. They are usually located on the extremities but rarely can be intraabdominal. Total surgical excision is usually curative if the lesion is symptomatic. The differential diagnosis of lipomas and other soft tissue tumors is very important to prevent possible complications and metastasis. CT and MRI are often used for this purpose, but pathological examination must be done for exact diagnosis.

\section{CASE REPORT}

A 49-year-old patient with abdominal pain and a palpable mass lesion was admitted to the clinic. Physical examination revealed a 20-cm-diameter giant, mobile, and firm mass lesion. CT examination showed a centrally located mesenteric, encapsulated, fat- density mass lesion that absorbed the contrast agent. Its size was about $190 \times 95 \mathrm{~mm}^{2}$, and differential diagnosis included lipoma and liposarcoma. Several lymph nodes were located peripheral to this lesion, the biggest one of them sized about $7.5 \mathrm{~mm}$ (Figure 1).

A decision regarding surgical exploration was made, and in the operation, a $15-\mathrm{cm}$ lesion was located $120 \mathrm{~cm}$ distal to the ligament of Trietz. The lesion was firm, mobile, and smooth surfaced. It involved the mesentery, but it was not derived from the intestine (Figure 2). Total excision with segmental resection was performed, followed by primary anastomosis after $30 \mathrm{~cm}$ of the resection (Figure 3). The patient discharged gas and stool on the third postoperative day. He was discharged from the hospital on the fourth postoperative day. Pathological diagnosis included mesenteric lipoma and reactive lymph nodes. No complications and complaints were reported at 1-month follow-up. 


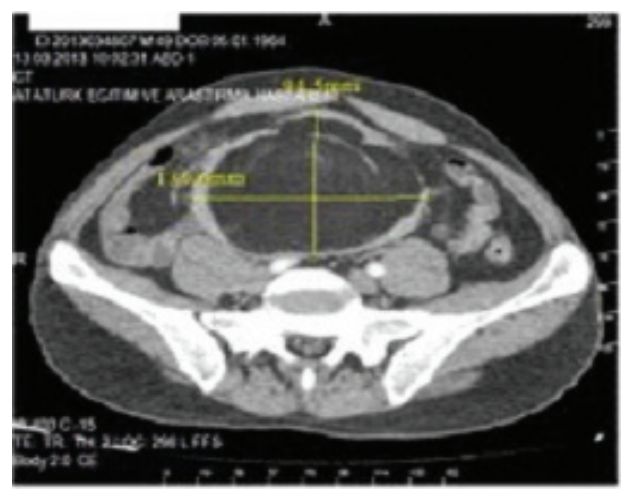

FIGURE 1

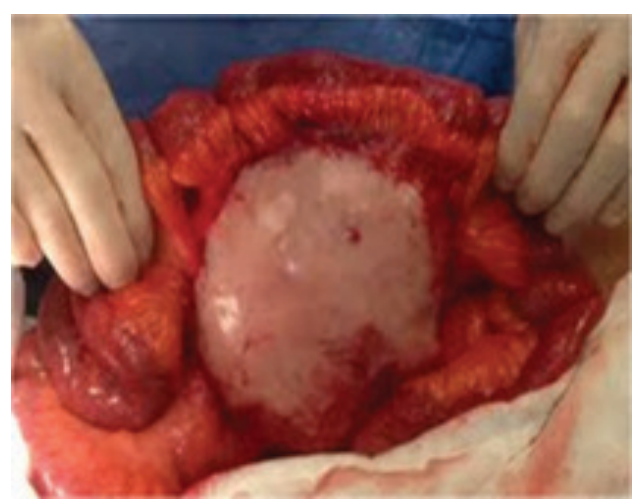

FIGURE 2

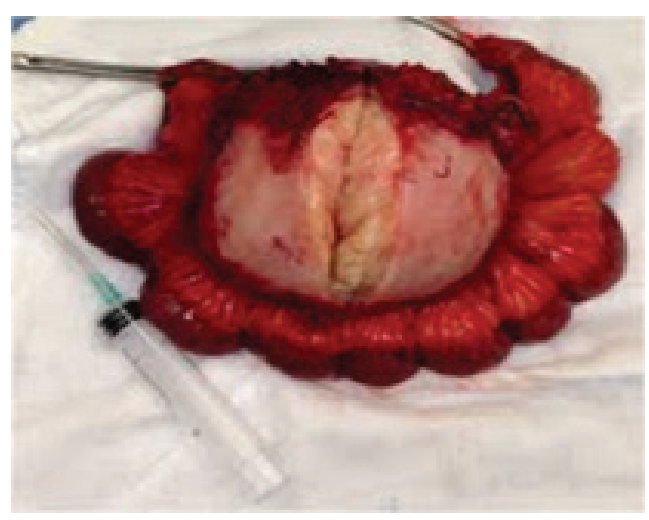

FIGURE 3

\section{DISCUSSION}

Lipomas, which are benign soft tissue tumors, can be seen on the extremities and in the mediastinum, retroperitoneum, and pelvis, but they are rarely intra-abdominal. They are nonlobulated, slowgrowing, nonsymptomatic, nonpenetrating mobile lesions that can cause abdominal pain, obstruction, distension, constipation, or acute abdominal symtoms based on their locations (24). They can incidentally be found during other nonrelevant or routine examinations. Mesenteric lipomas should not be forgotten during the differential diagnosis of intra-abdominal mass lesions. Liposarcomas, angiolipomas, teratomas, and such other soft tissue tumors should be considered when making differential diagnosis. In this case, imaging techniques could not differentiate between lipoma and liposarcoma. Liposarcomas are malignant fat tissue tumors. Ther are commonly seen in the fifth and sixth decades. They are mostly located in deep soft tissue and visceral regions. The prognosis depends mostly on the histological subtype of the tumor. Many subtypes of liposarcomas have been described. The aggresive ones are prone to cause lung metastasis and recur after excision (1).

The differentiation between lipoma and liposarcoma is very important. Since noninvasive techniques are not sufficient for this differentiation, surgical exploration has gained importance. Different types of symptoms can be seen according to the size and location of the tumor. The complications of mesenteric lipomas during childhood and adulthood have been reported. A study by Watt et al. found less than 50 cases in the English literature. In the same study, complications of an intestinal perforation were reported at the age of 72 , which led to a reoperation, $30-\mathrm{cm}$ intestinal resection, and ileostomization (5). The study by Colović et al. reported that surgical treatment was necessary for intestinal volvulus as a complication of mesenteric lipoma at the age of 77 (6). A study by Shah et al. reported that after intestinal resection for necrosis caused by intestinal torsion, surgical exploration was found to be curative in short bowel syndrome (3). On the contrary, the study by Fahri Halit Beşir et al. found that in one of the two patients with mesenteric lipoma, who were admitted to the hospital with abdominal pain, the symptoms could not be cured despite surgical treatment. Surgery is performed to make the diagnosis in some cases while to cure the acute or chronic symptoms in others. Good outcomes are usually achieved but surgery cannot be curative in all cases (8). 


\section{CONCLUSION}

Differential diagnosis is very important in intra-abdominal mass lesions. Imaging techniques are usually diagnostic, but in some cases, they cannot give exact results. Mesenteric lipomas, which are benign lesions, can cause chronic pain, obstruction, perforation, volvulus, and other such symptoms, as in the present study. Surgical exploration is needed for both making the differential daignosis and curing the symptoms.

\section{REFERENCES}

1. Robbins Basic Pathology 8th Turkish language edition Chapter 21 p 832.

2. Ozel SK, Apak S, Ozercan IH, Kazez A Giant mesenteric lipoma as a rare cause of ileus in a child: report of. Surg Today. 2004; 34(5): 470-2.
3. Sheen AJ, Drake I, George PP. A small bowel volvulus caused by a mesenteric lipoma: report of a case. Surg Today 2003; 33(8): 617-9.

4. Cherian A, Singh SJ, Broderick N, Zaitoun AM, Kapila L. Small bowel volvulus due to giant mesenteric lipoma. Pediatr Surg Int 2004; 20(11-12): 869-71

5. Watt DG, Sanjay P, Walsh SV, Young JA, Alijani A. Mesenteric lipoma causing small bowel perforation: a case report and review of literature. Scott Med J 2012; 57(4): 247

6. Colović R, Colović N. Mesenteric lipoma causing volvulus of the small intestine. Srp Arh Celok Lek 2000; 128(5-6): 205-7.

7. Shah SA, Saaiq M, Waqar SH, Rashid R, Jamal S. Mesenteric lipoma leading to small gut strangulation and short gut syndrome. J Coll Physicians Surg Pak 2005; 15(6): 371-2.

8. Beşir FH, Gül M, Koçak E, Karakaya K, Özdemir H, Gündoğdu S. Giant mesenteric lipoma caused abdominal pain: Report of two cases J Clin Exp Invest 2011; 2 (1): 95-97. 